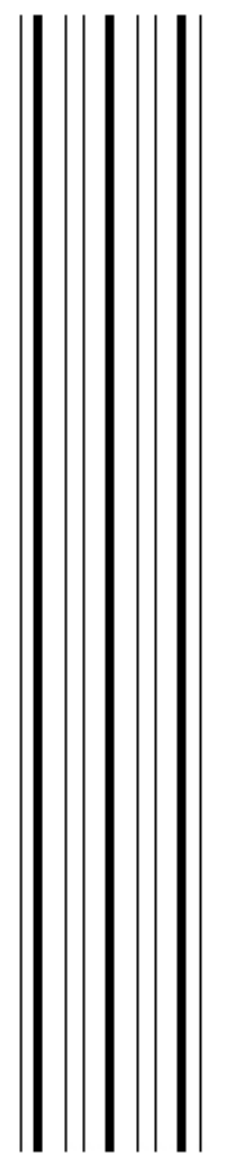

U.S. Department of Energy

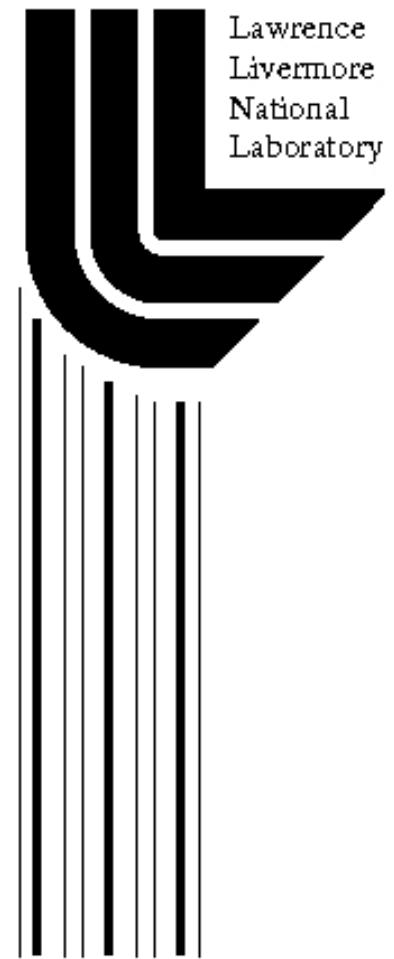

Doppler Broadening Update: Broadening near the Unresolved Resonance Region

\author{
by \\ Dermott E. Cullen \\ Lawrence Livermore National Laboratory \\ P.O.Box 808/L-198 \\ Livermore, CA 94550
}

January 7, 2012

\author{
Approved for public release; further dissemination unlimited
}




\section{DISCLAIMER}

This document was prepared as an account of work sponsored by an agency of the United States government. Neither the United States government nor Lawrence Livermore National Security, LLC, nor any of their employees makes any warranty, expressed or implied, or assumes any legal liability or responsibility for the accuracy, completeness, or usefulness of any information, apparatus, product, or process disclosed, or represents that its use would not infringe privately owned rights. Reference herein to any specific commercial product, process, or service by trade name, trademark, manufacturer, or otherwise does not necessarily constitute or imply its endorsement, recommendation, or favoring by the United States government or Lawrence Livermore National Security, LLC. The views and opinions of authors expressed herein do not necessarily state or reflect those of the United States government or Lawrence Livermore National Security, LLC, and shall not be used for advertising or product endorsement purposes.

This work performed under the auspices of the U.S. Department of Energy by Lawrence Livermore National Laboratory under Contract DE-AC52-07NA27344. 


\title{
Doppler Broadening Update: \\ Broadening near the Unresolved Resonance Region
}

\author{
by \\ Dermott E. Cullen \\ Lawrence Livermore National Laboratory \\ P.O.Box 808/L-198 \\ Livermore, CA 94550
}

January 7, 2012 


\begin{abstract}
Today when nuclear data codes use my SIGMA1 Doppler broadening method, generally we expect excellent agreement between the results produced by various nuclear data processing codes. Typically we see agreement to within roughly $0.1 \%$, which is much better than the accuracy to which we actually know the cross sections, i.e., broadening does not significantly increase the uncertainty in the cross sections. So it may raise concern when code users see large differences near the resolved unresolved resonance region energy boundary.
\end{abstract}

Today we see this difference between the results produced by various nuclear data processing codes, because in principle all of the codes mentioned here use my SIGMA1 method, but this method has been significantly updated since I originally published it, and each code may use a slightly different form of the method.

In this paper I explain the source of these differences and what various codes actually do today. Most important I hope to reassure code users that although I would personally prefer that all processing codes use the most current version of my SIGMA1 method, in terms of integral effects on overall system parameters we expect little or no macroscopic effect, and code users should not be overly concerned by such differences.

\title{
Introduction
}

My SIGMA1 method of Doppler broadening [1, 2] addressed the problem of Doppler broadening cross sections that are tabulated and linearly interpolable between tabulated values; starting from these tabulated cross sections in principle the SIGMA1 method produces exact Doppler broadened cross sections.

This method was developed to be applied to tabulated cross sections in the ENDF format [3]. The method is used in many nuclear data processing codes $[4,5,6]$. The method has been in use for almost 40 years, and by now it has become the standard method for Doppler broadening throughout the World. 
Since its ORIGINAL definition [1, 2] the SIGMA1 method has been significantly improved based primarily on many hundreds of man-years of experience by many code users throughout the World. I recently documented the CURRENT SIGMA1 method in the Handbook of Nuclear Engineering [7] as used in my PREPRO codes [4]. Here I provide more information and examples of the use of the SIGMA1 method with evaluations that include an unresolved resonance region [3].

\section{A Brief Review of Doppler broadening}

We Doppler broaden cross sections to account for the thermal motion of target nuclei, that can change the relative speed between our projectile (e.g., a neutron) and target nuclei (e.g., U235), which changes the reaction rates in our systems. We Doppler broaden starting from known cross sections at a temperature $\mathrm{T} 1, \sigma(E, T 1)$, and define cross sections at a higher temperature $\mathrm{T} 2, \sigma(E, T 2)$. Using speed $(\mathrm{V})$, rather than energy $(\mathrm{E})$, the equation to solve is,

$$
\begin{aligned}
& V \sigma(V, T 2)=\frac{1}{V}\left(\frac{\beta}{\pi}\right){ }^{1 / 2} \int_{0}^{\infty}[V r \sigma(V r, T 1)] V r d V r \times \\
&\left\{\operatorname{Exp}\left[-\beta(V-V r)^{2}\right]-\operatorname{Exp}\left[-\beta(V+V r)^{2}\right]\right\}
\end{aligned}
$$

$\beta=\frac{m}{2 K(T 2-T 1)}$

It can be easily shown [2] that this is merely an integral form of the diffusion equation in spherical coordinates (velocity space), where the variable becoming "smooth" is the reaction rate [speed times cross section; $v *$ $\sigma(E, T 2)]$. The SIGMA1 method of Doppler broadening assumes that the initial cross section, $\sigma(E, T 1)$, is defined by tabulated, linearly interpolable cross sections. With this assumption in principle the Doppler broadening equation can be "exactly" solved with no further assumptions. 


\section{Starting Cross Sections}

One driving force behind developing the SIGMA1 method was the fact that at the time a number of codes had already been developed to produce tabulated, linearly interpolable cross sections in the ENDF format. To complement this "cold" data I wanted to develop a method to produce "hot" (Doppler broadened) data, also in the ENDF format, so that our codes could use it exactly as they used "cold" data.

These codes started from a combination of originally tabulated data and added any resonance contribution, to in principle produce data over the entire energy range, in exactly the form needed as input to the SIGMA1 method; SIGMA1 could start from this "cold" data (at T1) and produce Doppler broadened cross sections (at T2), and output the results in the ENDF format. I say in principle, because what was overlooked in the ORIGINAL SIGMA1 method is that in the unresolved resonance region (URR) the "cold" data output by these codes are tabulated, infinitely dilute "average" values. By definition infinitely dilute "averages" are temperature INDEPENDENT. So that Doppler broadening should not change these values. However, the ORIGINAL SIGMA1 method treated the tabulated "averages" as if they were energy dependent values, and Doppler broadened them. The CURRENT SIGMA1 corrects this problem; explaining this point and the effects that it has is the primary purpose of this paper.

\section{Explanation of Figures}

In the appendix I include figures to illustrate the effect on cross sections near the unresolved resonance region (URR) boundaries using the CURRENT versus the ORIGINAL SIGMA1 method. All of the figures were produced using the CURRENT SIGMA1 code (part of PREPRO 2012); the CURRENT code has an option to treat the unresolved resonance region using either the CURRENT or ORIGINAL method.

There are a variety of differences between the ORIGINAL SIGMA1 method, circa 1972, and the CURRENT method, but it is important that the reader understand that the differences in the figures included in this report are ONLY due to the treatment of the URR. Today there may be other sources of differences between the results produced by various codes, due to differences in the version of SIGMA1 they use, as well as other approximations they may use. I judge that trying to cover all of these 
differences is beyond the scope of this paper, so that here I only cover differences due to the handling of the unresolved resonance region (URR); this is the source of differences most obvious when we compare code results.

\section{The Effect of Doppler Broadening}

Since Doppler broadening can affect the reaction rates in our system it is very important to accurately account for temperature dependent effects. For cross sections that include resonances the effect can extend to higher energies than you might think. . For example, the resolved resonance region of ENDF/B-VII U235 extends up to $2.25 \mathrm{keV}$, and for U238 up to $20 \mathrm{keV}$. In the appendix I compare "cold", 0 Kelvin, and "room temperature", 293.6 Kelvin, cross sections for U235 and U238. The figures in the appendix illustrate that even "room temperature", $293.6 \mathrm{~K}(0.0253 \mathrm{eV}$, roughly $1 / 40$ $\mathrm{eV}$ ) has a significant effect on the energy dependent cross sections well into the $\mathrm{keV}$ energy range.

All of the figures in the appendix include comparisons of total, elastic, capture and fission cross sections. The upper two thirds of each plot compares cross sections, and the lower third of each plot shows the ratio of the cross sections.

Fig. 1 and 2 compare U235, $0 \mathrm{~K}$ and $293.6 \mathrm{~K}$ cross sections near the resolved - unresolved resonance region boundary at $2.25 \mathrm{keV}$. Fig 1 covers the energy range 2.20 to $2.26 \mathrm{keV}$, to give us a view of a broader range of energies and resonances. Fig 2 covers the narrower energy range 2.24 to 2.26 , to give us a more detailed view near the $2.25 \mathrm{keV}$ interface.

Similarly, Fig. 5 and 6 compare U238, $0 \mathrm{~K}$ and $293.6 \mathrm{~K}$ cross sections near the resolved - unresolved resonance region boundary at $20 \mathrm{keV}$. Fig 5 covers the energy range 19.6 to $20.1 \mathrm{keV}$, to give us a view of a broader range of energies and resonances. Fig 6 covers the narrower energy range 19.9 to 20.1 , to give us a more detailed view near the $22 \mathrm{keV}$ interface.

The important point to note for $\mathbf{U} 235$ (figs. 1 and 2), and $\mathbf{U 2 3 8}$ (figs. 5 and 6), is the strong effect that Doppler broadening has; even room temperature drastically changes the cross sections well into the keV energy range; this effect is particularly obvious for the narrow capture resonances shown in these figures. 


\section{Solution: Where the SIGMA1 Method Works}

The SIGMA1 method is designed to start from tabulated, linearly interpolable cross section. These can be obtained from current ENDF formatted [3] evaluations, by first processing the original evaluation to make all tabulated cross section linearly interpolable, and by adding the resonance parameter contribution; the result is tabulated, linearly interpolable cross sections.

The SIGMA1 method starts from tabulated, linearly interpolated cross sections and the cross sections included in evaluations are only tabulated over a finite energy range; for $\mathrm{ENDF} / \mathrm{B}$ evaluations most cross sections extend from a lower energy of $10^{-5} \mathrm{eV}$ up to at least $20 \mathrm{MeV}$. The Doppler broadening equation involves an integral over ALL energies. Therefore to use the SIGMA1 method over this entire energy range we define the reaction rate [speed times cross section; $v * \sigma(E)$ ] outside of the tabulated energy range to be constant, equal to its value at the nearest tabulated, energy dependent energy. Since this is an important concept let me restate it: Below $10^{-5} \mathrm{eV}$ down to zero energy we extend the reaction rate $[v * \sigma(E)]$ as constant, equal to its value at $10^{-5} \mathrm{eV}$; similarly above $20 \mathrm{MeV}$ up to infinity we define the reaction rate $[v * \sigma(E)]$ to be constant, equal to its value at $20 \mathrm{MeV}$.

One point to understand: in my brief summary of Doppler broadening I mentioned that the Doppler broadening equation "smooth" the reaction rate [speed times cross section; $v * \sigma(E)$ ]. My decision to extend the reaction rate as constant outside the tabulated energy range of the cross section is to minimize end effects; at low energy this is particularly important, because it insures that $1 / \mathrm{v}$ cross sections (constant reaction rate) is temperature dependent.

Today many processing codes $[4,5,6]$ use this approach to prepare temperature dependent tabulated cross sections for later use in applications. This approach is straight forward for cross sections that are originally tabulated using linear or non-linear interpolation, and/or resolved resonance parameters. 


\section{Problem: Where the SIGMA1 Method does not Work}

The problem is that in the unresolved resonance region (URR), by definition, we do not know the energy dependent cross sections; we only know average values and the distribution of cross sections. As such the SIGMA1 method should not be applied to the unresolved resonance region.

In a recent paper I discussed the problem of consistently defining the average cross section INSIDE the unresolved resonance region [8], using methods that are appropriate for use in the URR. Based on that paper we now have general agreement between major data processing codes $[4,5,6]$; so that we now consider that problem to be solved. Let me stress this point: today we feel that the problem INSIDE the URR has been solved [8], and here we need only be concerned with cross sections OUTSIDE, and near the boundaries of the URR.

In this paper I discuss how to define the energy dependent cross section OUTSIDE of, but near the unresolved resonance region. Doppler broadening involves solving an integral equation, where in order to define the cross section at any energy, E, we MUST define "cold" cross sections at all energies. In principle this integral extends from zero energy to infinite energy. In practice contributions to the integral really only extends over an energy interval near the energy $\mathrm{E}$; it extends both below and above the energy $\mathrm{E}$.

\section{What's the Solution?}

The ORIGINAL SIGMA1 method [1,2] did not consider the average cross sections in the unresolved resonance region (it treated them as tabulated, energy dependent data), so that once we extended the tabulated data down to zero energy and up down infinity we could proceed to Doppler broaden all cross sections over the entire energy range. Since the cross sections in the unresolved region are average values, rather than energy dependent values needed by the SIGMA1 method, this was not correct.

The CURRENT SIGMA1 method $[4,7]$ more correctly treats the unresolved resonance region by,

1) Copying the infinitely dilute average values within the URR without change; this relies on the definition of the infinitely dilute average as 
being temperature independent. This allows subsequent processing to correctly include self-shielding in the unresolved region [7].

2) Extending the tabulated, linearly interpolable energy dependent data outside the unresolved region into the unresolved region as a constant reaction rate [speed times cross section; $v * \sigma(E)$ ], exactly in the same manner as the original method extended the data outside of its tabulated range. As explained above, extending the reaction rate as constant equal to its tabulated value OUTSIDE the URR will minimize changes near the boundary.

WARNING - Often at the resolved - unresolved boundary there will be a discontinuity in the cross section, with a repeated energy exactly at the boundary; one value corresponds to the last tabulated energy dependent cross section OUTSIDE the URR, and the second value corresponds to the first average value INSIDE the URR. Remember that the SIGMA1 method can only be used with tabulated, energy dependent cross sections. So it is important when extending the reaction rate to use the tabulated value OUTSIDE, not the average value INSIDE the URR. See figs. 1 through 8 to appreciate the enormous differences that can exist between these two values.

When we compare the results using the ORIGINAL and CURRENT SIGMA1 methods, this naturally leads to differences in the cross sections near the boundaries of the unresolved resonance region.

\section{Differences between ORIGINAL and CURRENT SIGMA1}

To illustrate the differences in the results in the appendix I show results for U235 and U238 using the SIGMA1 method CURRENT (URR) treatment) and ORIGINAL (No URR) treatment. In each case I show results near the resolved - unresolved region boundary.

Fig. 3 and 4 compare U235, CURRENT (URR) and ORIGINAL (No URR) cross sections near the resolved - unresolved resonance region boundary at $2.25 \mathrm{keV}$. Fig 3 covers the energy range 2.20 to $2.26 \mathrm{keV}$, to give us a view of a broader range of energies and resonances. Fig 4 covers the narrower energy range 2.24 to 2.26 , to give us a more detailed view near the $2.25 \mathrm{keV}$ interface. 
Similarly, Fig. 7 and 8 compare U238, CURRENT (URR) and ORIGINAL (No URR) cross sections near the resolved - unresolved resonance region boundary at $20 \mathrm{keV}$. Fig 7 covers the energy range 19.6 to $20.1 \mathrm{keV}$, to give us a view of a broader range of energies and resonances. Fig 8 covers the narrower energy range 19.9 to 20.1 , to give us a more detailed view near the $22 \mathrm{keV}$ interface.

The first point to note is that the CURRENT method minimizes changes in the reaction rate near the interface, whereas the ORIGINAL method results in large changes. This is because almost all evaluations end their resolved resonance region in a minimum between resonances, so that extending the reaction rate based on the last tabulated energy dependent cross section OUTSIDE into the URR, results in smooth changes with temperature. In contrast the ORIGINAL method uses the average points INSIDE the URR, which makes it appear that there is a continuous string of strong resonances immediately adjacent to the resolved region. The result is an abrupt increase in the cross section just OUTSIDE the URR and a decrease in the cross section just INSIDE the URR; this effect can be seen most easily in figs. 4 and 8.

The most important point to note from Figs. 3, 4, 7, and 8, is that the two results are in excellent agreement over most of the resolved resonance region, and differ only over a relatively small energy range near the resolved - unresolved resonance region interface. Near the interface the differences can be enormous, but only over such small energy ranges that we expect little effect on the overall integral system parameters.

\section{Conclusion}

Today when nuclear data codes use my SIGMA1 Doppler broadening method $[1,2]$, generally we expect excellent agreement between the results produced by various nuclear data processing codes $[3,4,5]$. Typically we see agreement to within roughly $0.1 \%$, which is much better than the accuracy to which we actually know the cross sections, i.e., broadening does not significantly increase the uncertainty in the cross sections. So it may raise concern when code users see large differences near the resolved unresolved resonance region energy boundary.

Today we see this difference between the results produced by various nuclear data processing codes, because in principle all of the codes 
mentioned here use my SIGMA1 method, but this method has been significantly updated since I originally published it, and each code may use a slightly different form of the method. Here is a brief summary, so that users can understand what they should expect each code,

1) PREPRO [3] uses what I called the CURRENT method.

2) NJOY [4] uses a mix of ORIGINAL and CURRENT method.

3) AMPX [5] uses what I call the ORIGINAL method.

NJOY [4] uses a mix in the sense that it uses the ORIGINAL method to ignore the unresolved region and Doppler broaden the entire energy range. But it then uses the CURRENT method by replacing the energy points within the unresolved region by the original average, infinitely dilute, unbroadened cross sections. As a result the PREPRO and NJOY results agree INSIDE the unresolved region and differ only OUTSIDE near the resolved - unresolved resonance region boundary. In contrast the PREPRO and AMPX results differ both INSIDE and OUTSIDE the unresolved region as shown in figs. $3,4,7$ and 8 .

In this paper I explain the source of these differences and what various codes actually do today $[3,4,5]$. Most important I hope to reassure code users that although I would personally prefer that all processing codes use the most current version of my SIGMA1 method, in terms of integral effects on overall system parameters we expect little or no macroscopic effect, and code users should not be overly concerned by such differences.

\section{Acknowledgments}

I thank Robert MacFarlane (LANL/retired) and Maurice Greene (ORNL/retired) for supplying results from their codes, NJOY and AMPX, which helped me to understand the sources of the differences we see near the URR boundary. I thank the following for reviewing a preliminary version of this paper and making constructive criticism that has been incorporated in the final version of this paper: John Scorby (LLNL), Robert MacFarlane (LANL), Maurice Greene (ORNL), S. Ganesan (BARC), Andrej Trkov (IJS), Jean Christophe Sublet (CEA). 


\section{References}

[1] "Exact Doppler Broadening of Evaluated Neutron Cross Section," Trans. Amer. Nuc. Soc. 16, p. 320 (1973), by D.E. Cullen, etal.

[2] "Exact Doppler Broadening of Tabulated Cross Sections," Nuclear Science and Engineering 60, p. 199 (1975), by D.E. Cullen, etal.

[3] ENDF format: "ENDF-6 Formats Manual: Data Formats and Procedures for the Evaluated Nuclear Data File ENDF/B-VI and ENDF/BVII", CSEWG Document ENDF-102, edited by A. Trkov, M. Herman and D.A. Brown, (Nov. 2011). Note, that the ENDF formats and conventions have had a number of updates; the latest version is available on-line at, http://www.nndc.bnl.gov/exfor/4web/endf-manual-dec2011.pdf

In this paper I attempt to be consistent to distinguish between: ENDF which is a FORMAT, that almost all evaluated data libraries use today; and ENDF/B which is the U.S. DATA LIBRARY, which uses the ENDF format, similar to JEFF, JENDL, and other data libraries, throughout the World.

[4] PREPRO 2010: 2010 ENDF/B pre-processing Codes", IAEA-NDS-39, Rev. 14, October, 2010, by Dermott E. Cullen, Nuclear Data Section, International Atomic Energy Agency, Vienna, Austria. These codes are available FREE on-line at http://www-nds.iaea.or.at/ndspub/endf/prepro/

[5] NJOY: "The NJOY Nuclear Data Processing System, Version 91," Los Alamos National Laboratory report LA-12740-M, by R. E. MacFarlane and D. W. Muir, (October 1994) is still the latest official manual.

[6] "AMPX: A Modular Code System for Generating Coupled Multigroup Neutron-Gamma Libraries from ENDF/B", by N.M. Greene, et al., ORNLTM-3706, Oak Ridge National Laboratory, Oak Ridge, Tenn., 1976.

[7] "Nuclear Data Preparation", pp. 279-425, Vol. 1, in "The Handbook of Nuclear Engineering", Springer Publishing, NY, NY (2010), by D.E. Cullen, Edit D. Cacuci.

[8] "A Short History of ENDF/B Unresolved Resonance Parameters", LLNL-TR-461199, October 2010, by D.E. Cullen 
Fig. 1: U235 cross sections, “cold”, 0 K, and "room temperature”, 293.6 K
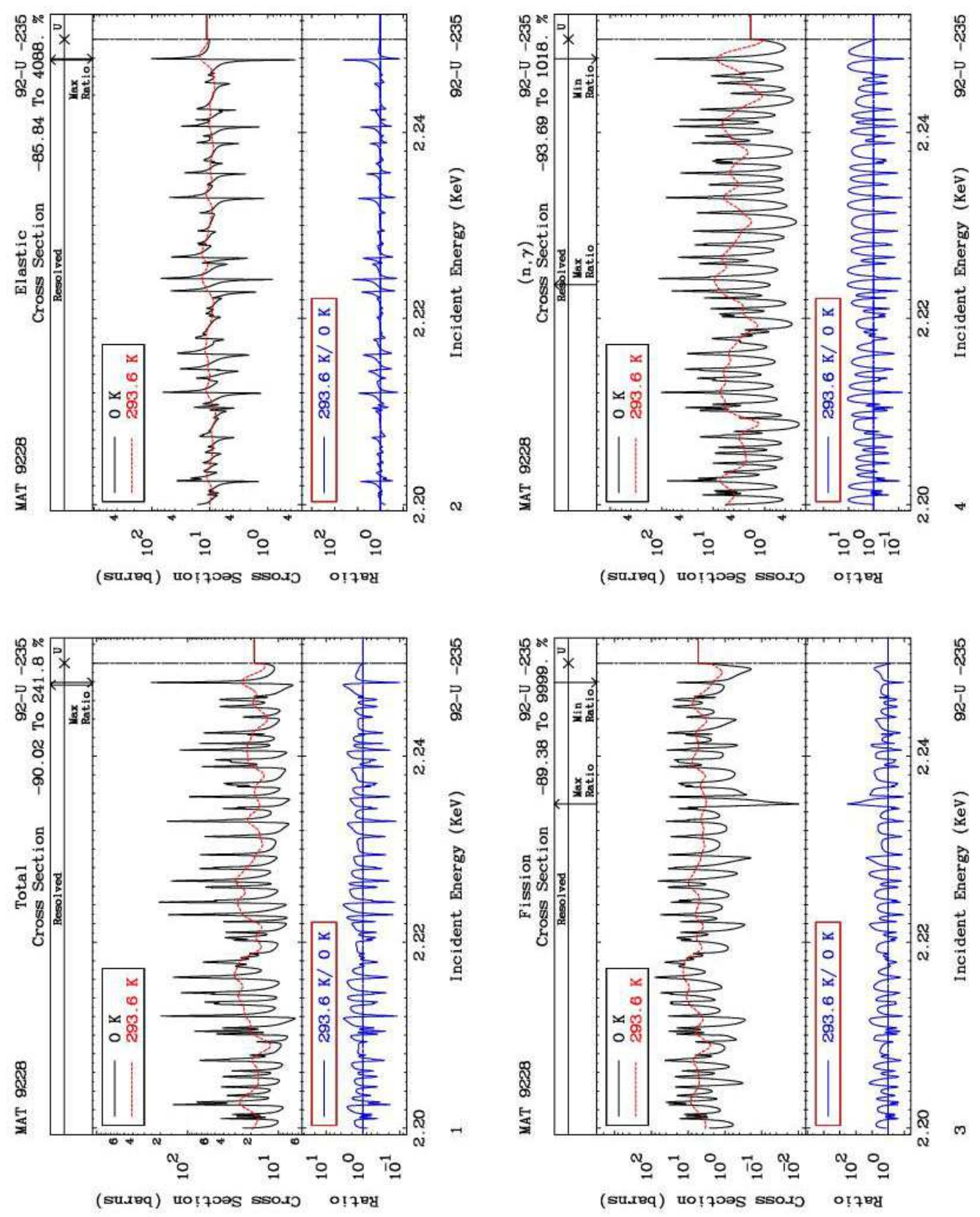
Fig. 2: U235 cross sections, “cold”, 0 K, and "room temperature”, 293.6 K
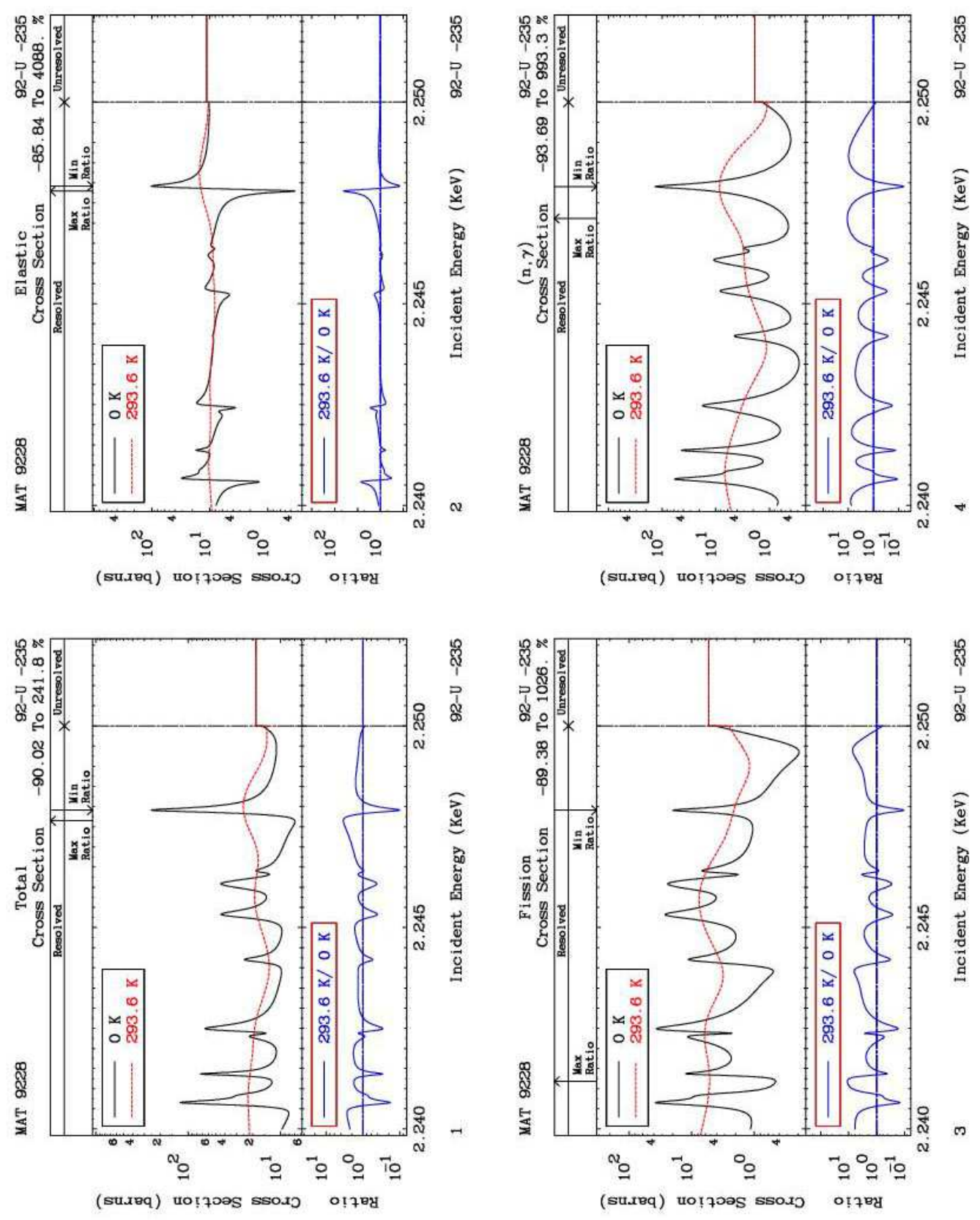
Fig. 3: U235 cross sections, with and without unresolved resonance region (URR)
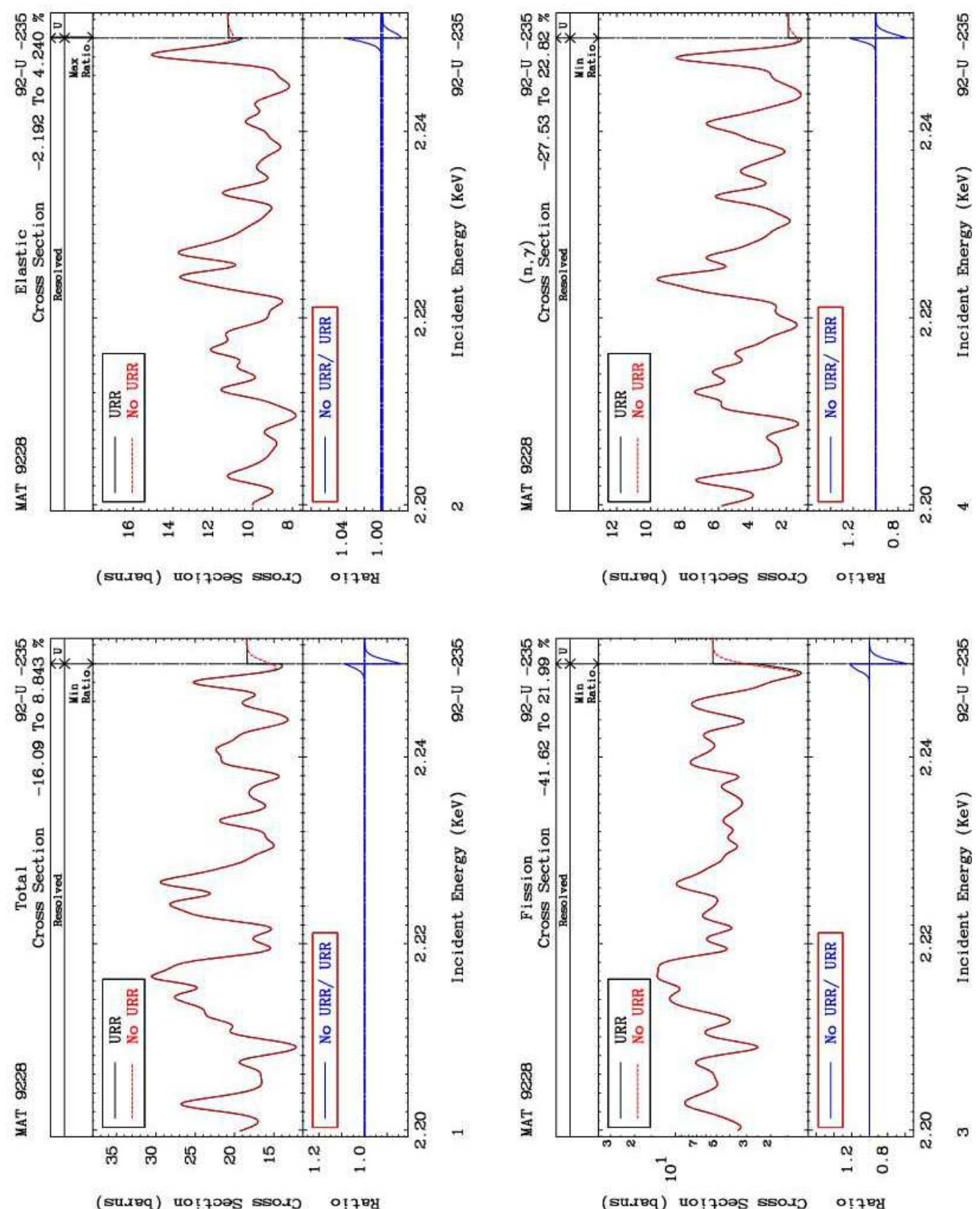
Fig. 4: U235 cross sections, with and without unresolved resonance region (URR)

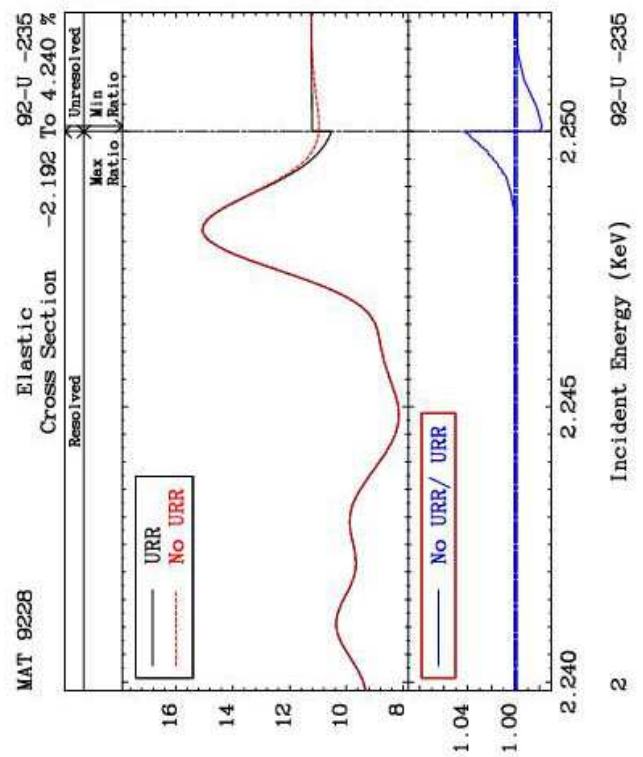

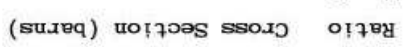

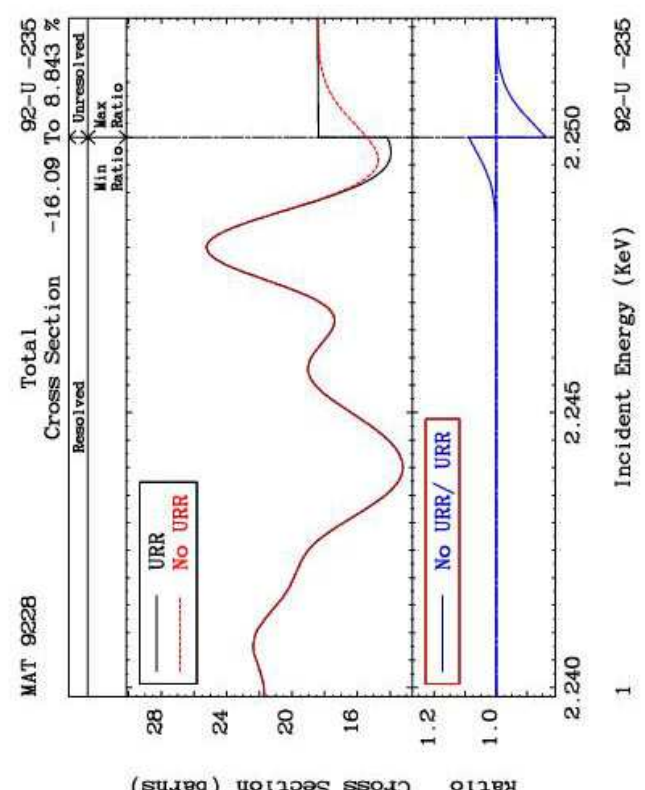

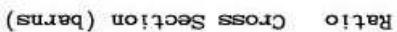

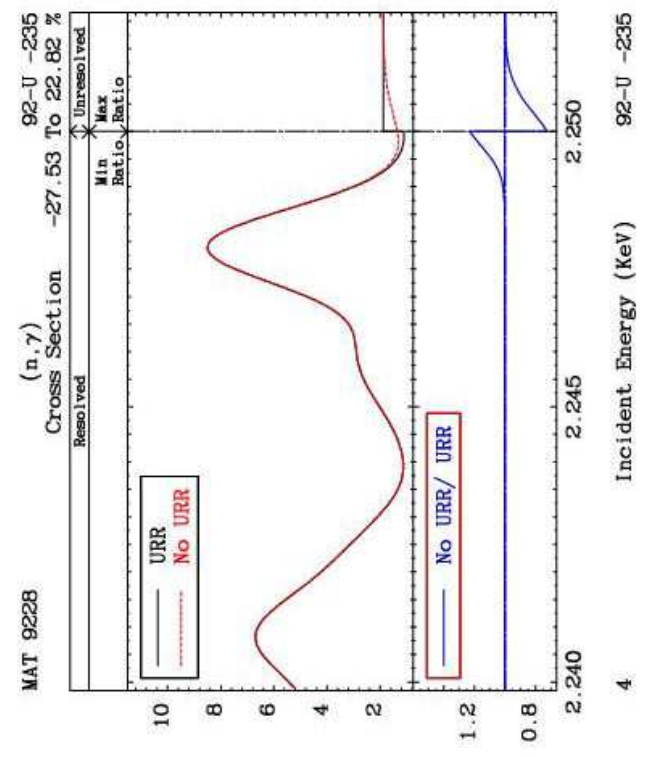

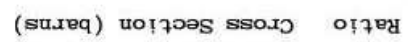

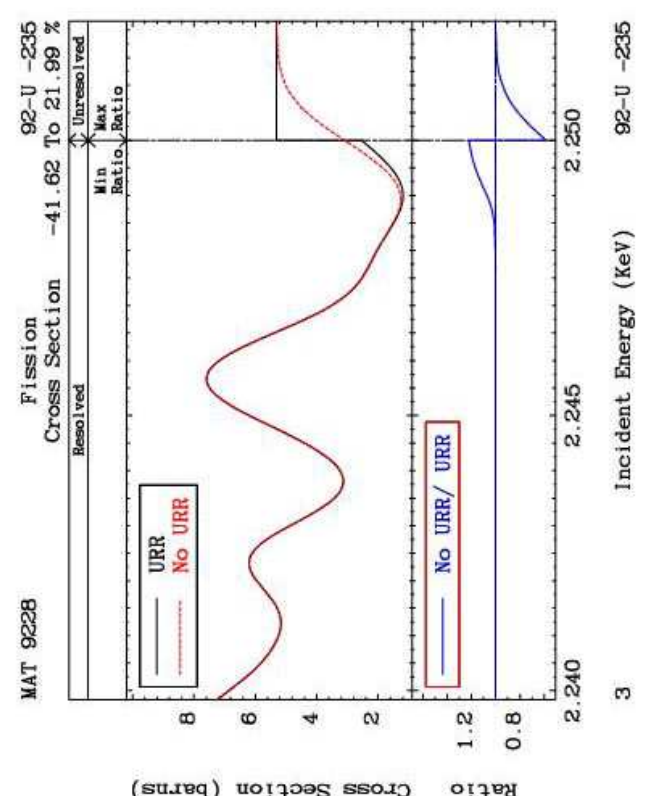

(su.req) uoț700s ssoxว отฺฺष 
Fig. 5: U238 cross sections, “cold”, 0 K, and "room temperature”, 293.6 K
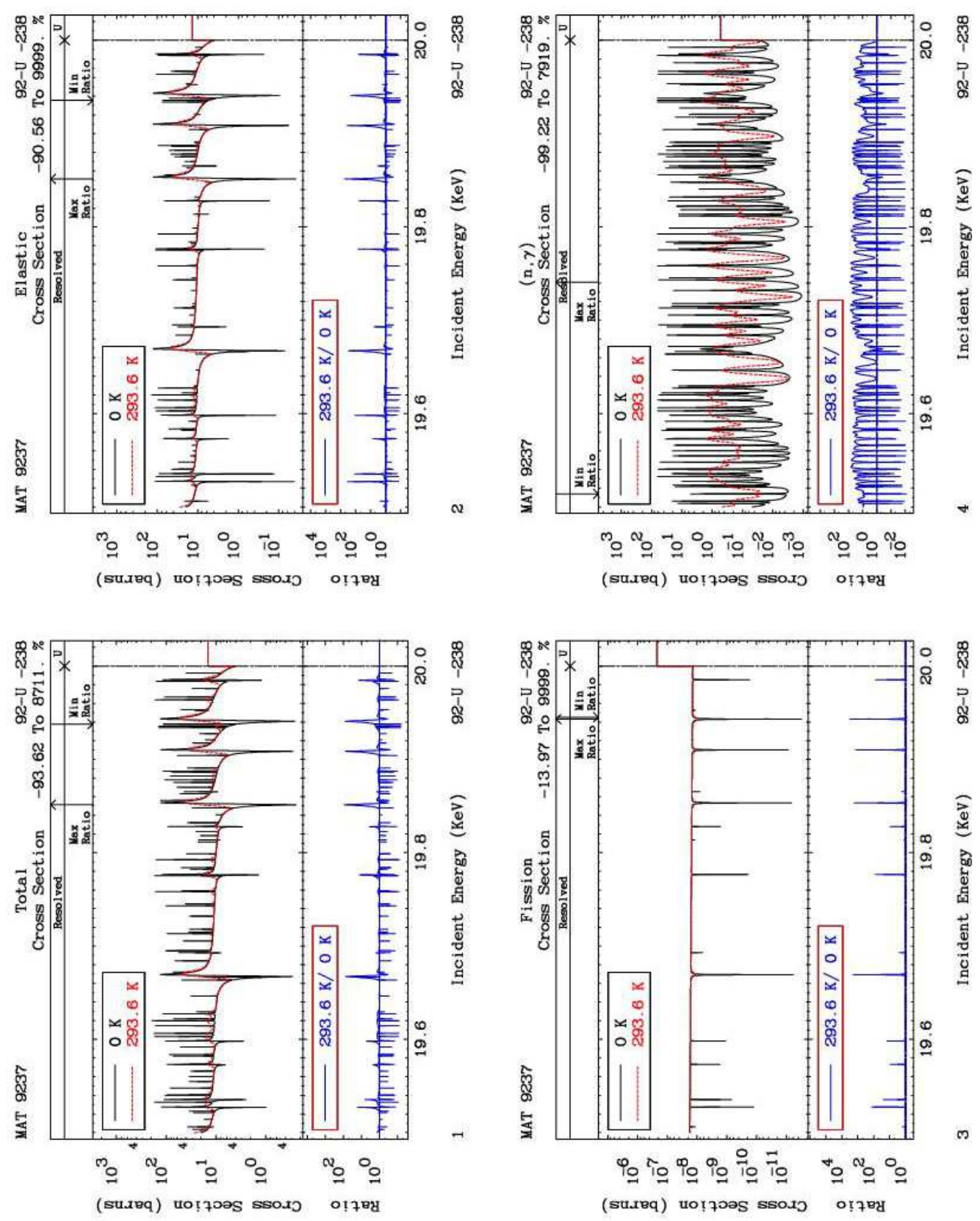
Fig. 6: U238 cross sections, “cold”, 0 K, and "room temperature”, 293.6 K
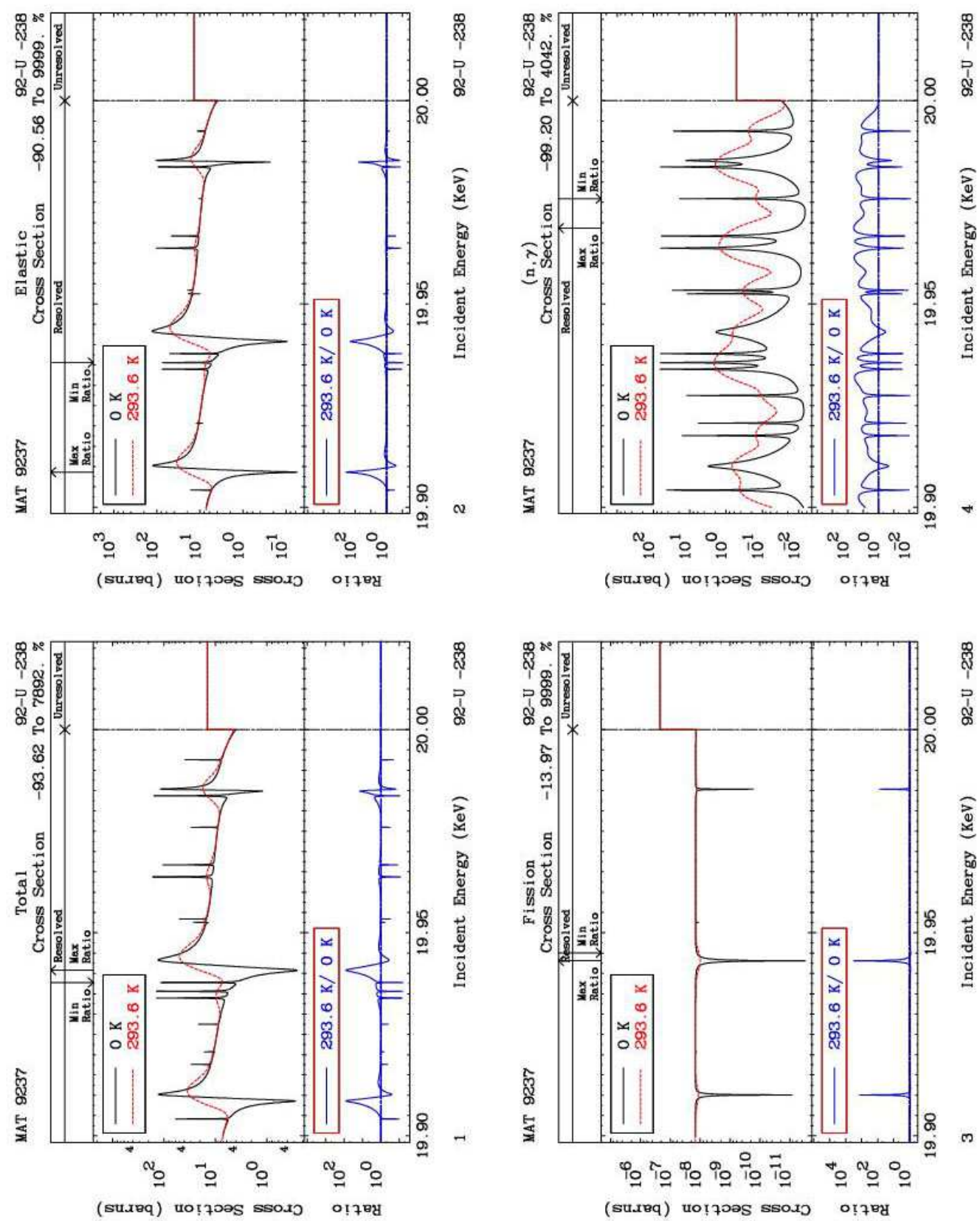
Fig. 7: U238 cross sections, with and without unresolved resonance region (URR)

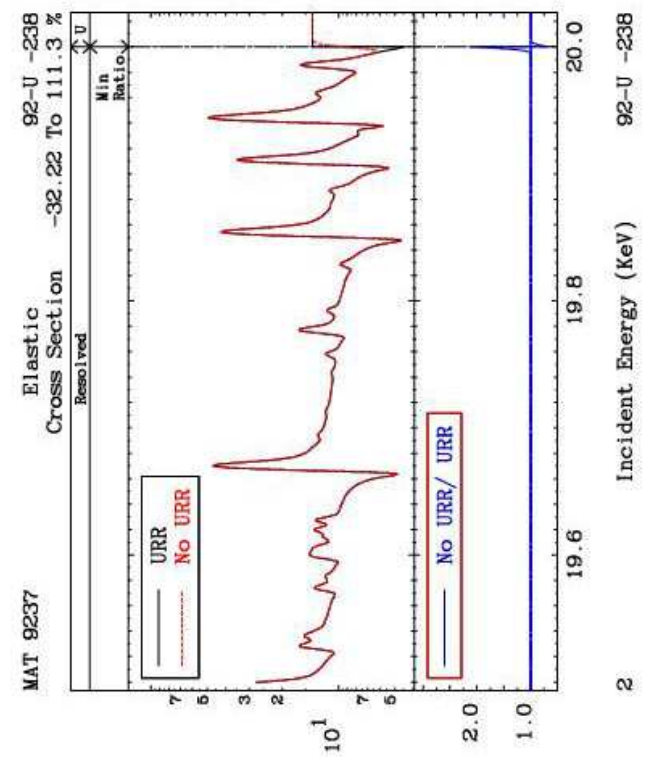

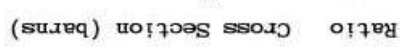
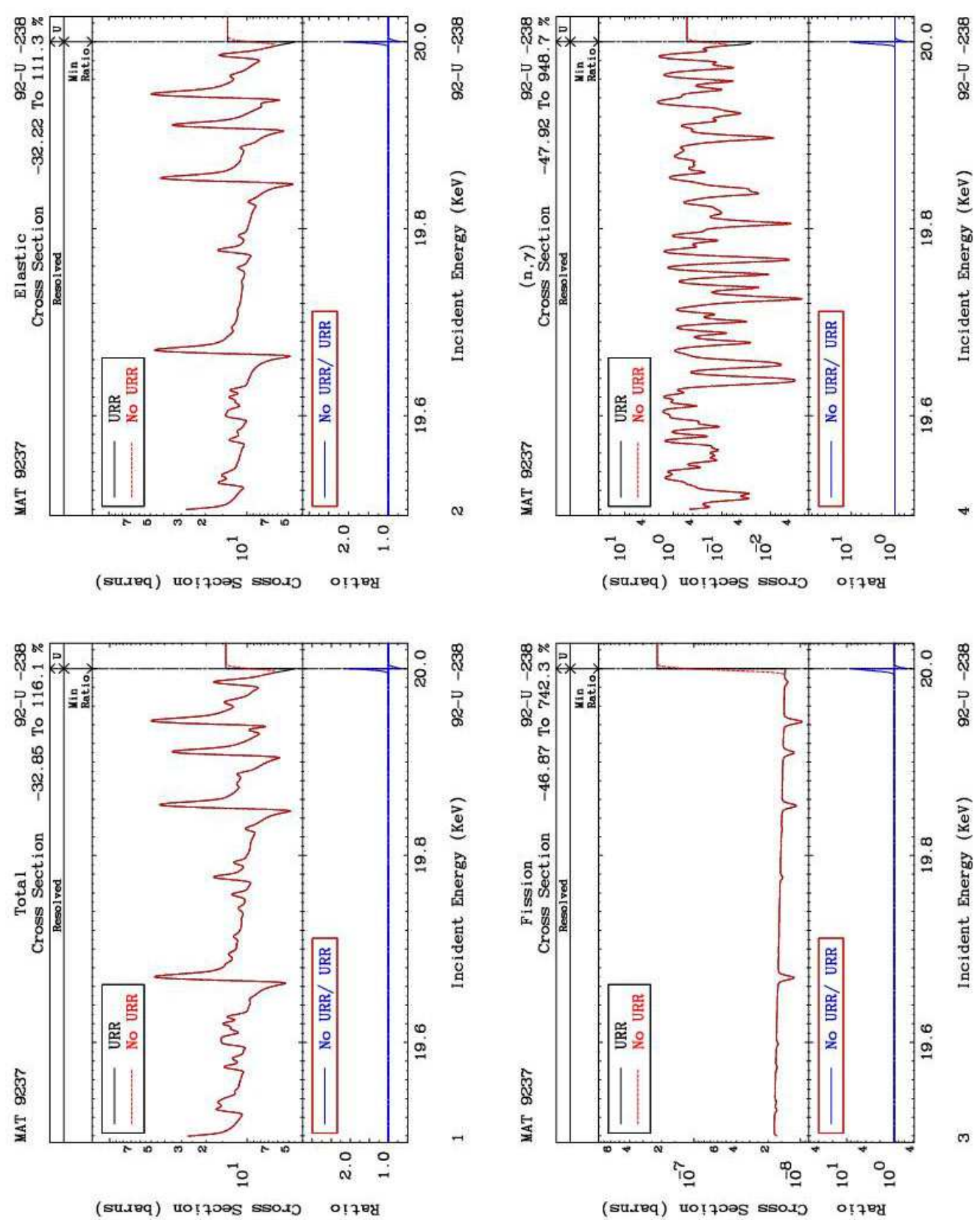
Fig. 8: U238 cross sections, with and without unresolved resonance region (URR)

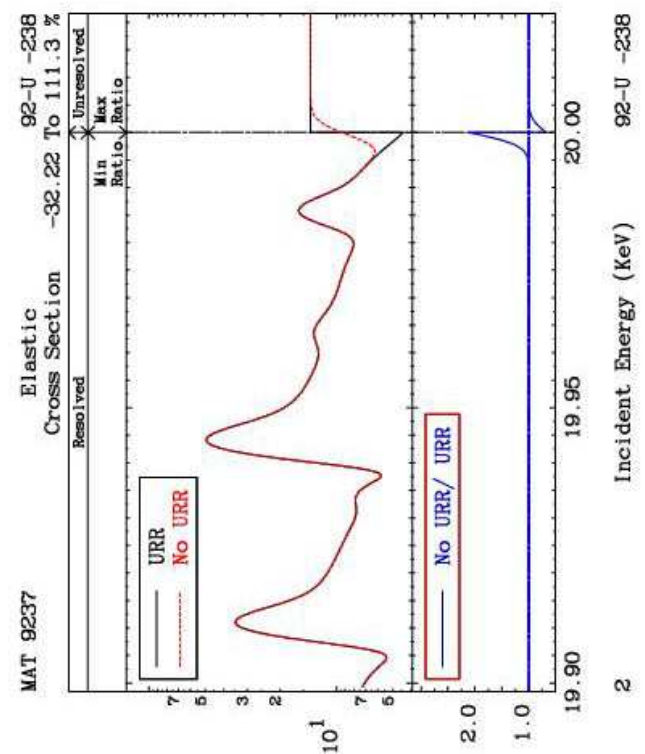

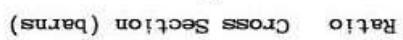

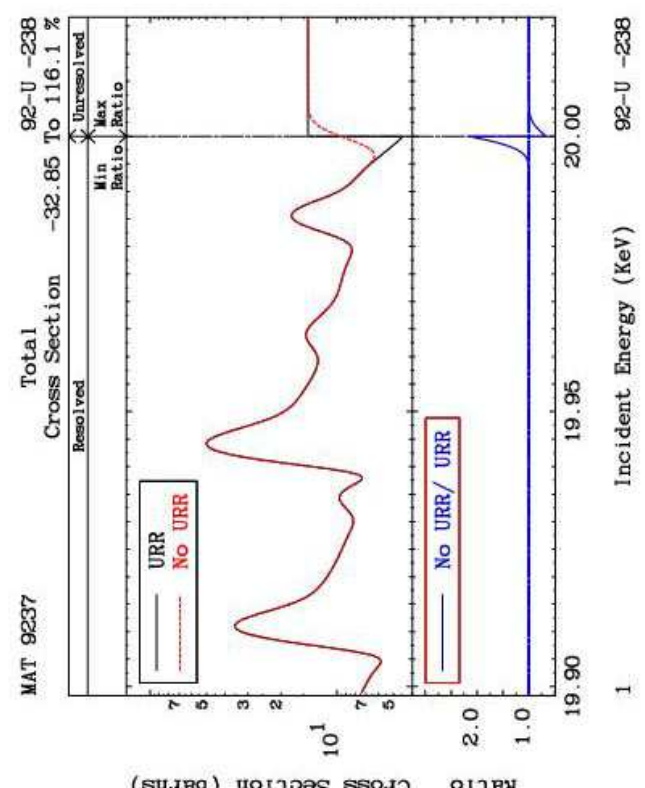

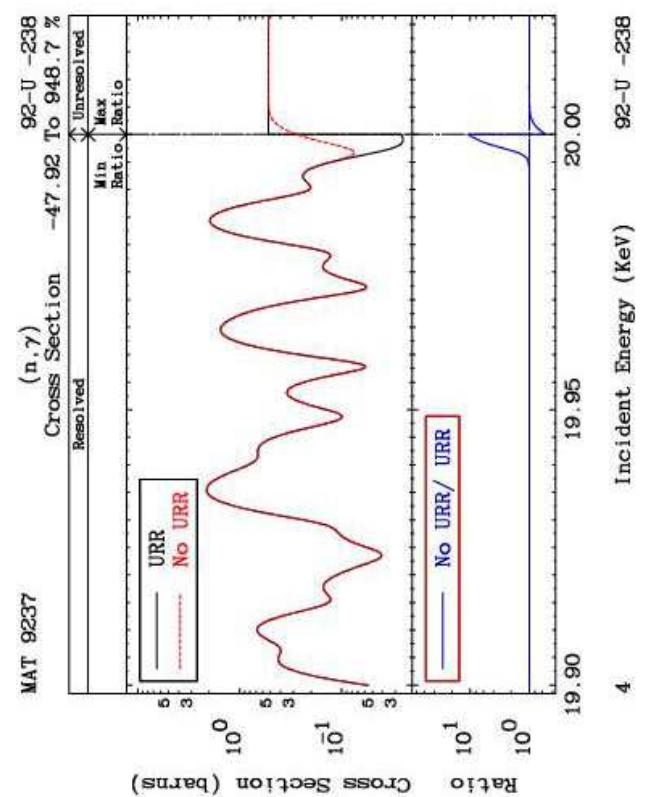

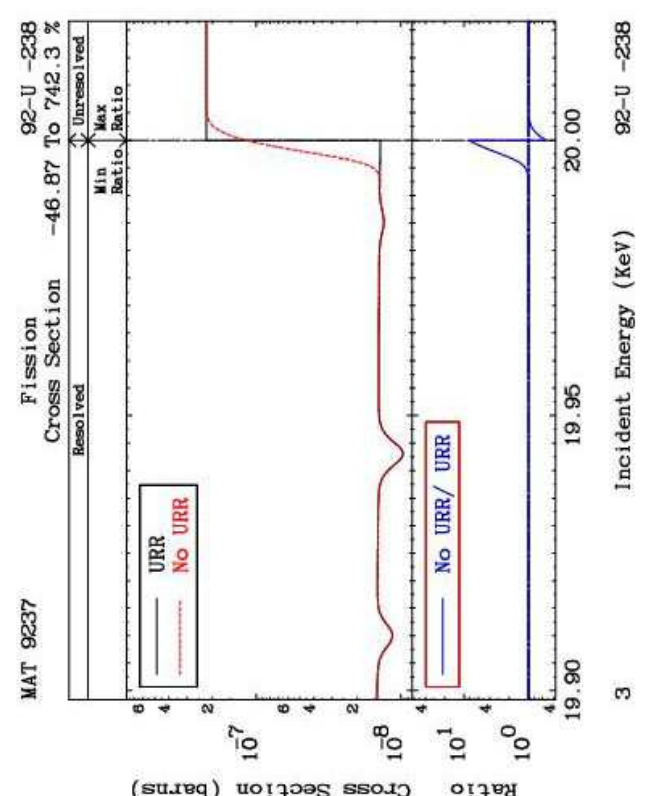

